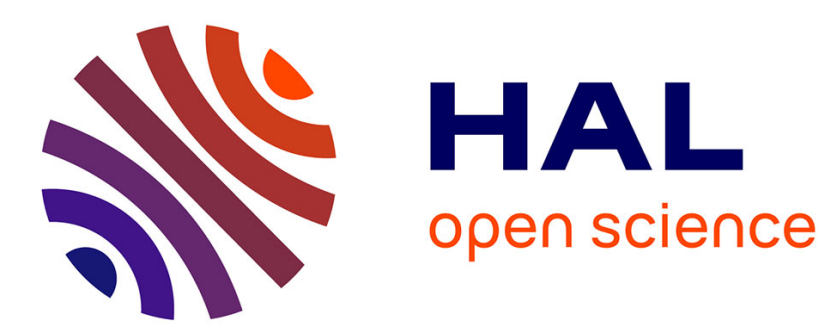

\title{
Higher-Order Leaky-Mode Bessel-Beam Launcher
}

Walter Fuscaldo, Guido Valerio, Alessandro Galli, Ronan Sauleau, Anthony

Grbic, Mauro Ettorre

\section{To cite this version:}

Walter Fuscaldo, Guido Valerio, Alessandro Galli, Ronan Sauleau, Anthony Grbic, et al.. HigherOrder Leaky-Mode Bessel-Beam Launcher. IEEE Transactions on Antennas and Propagation, 2016, 64 (3), pp.904-913. 10.1109/TAP.2015.2513076 . hal-01331289

\section{HAL Id: hal-01331289}

\section{https://hal-univ-rennes1.archives-ouvertes.fr/hal-01331289}

Submitted on 12 Mar 2020

HAL is a multi-disciplinary open access archive for the deposit and dissemination of scientific research documents, whether they are published or not. The documents may come from teaching and research institutions in France or abroad, or from public or private research centers.
L'archive ouverte pluridisciplinaire HAL, est destinée au dépôt et à la diffusion de documents scientifiques de niveau recherche, publiés ou non, émanant des établissements d'enseignement et de recherche français ou étrangers, des laboratoires publics ou privés. 


\title{
Higher-Order Leaky-Mode Bessel-Beam Launcher
}

\author{
Walter Fuscaldo, Student Member, IEEE, Guido Valerio, Member, IEEE, Alessandro Galli, Member, IEEE, \\ Ronan Sauleau, Senior Member, IEEE, Anthony Grbic, Senior Member, IEEE, and Mauro Ettorre, Senior \\ Member, IEEE
}

\begin{abstract}
We present a Bessel-beam launcher based on a leaky radial waveguide consisting of a capacitive sheet over a ground plane that supports higher-order leaky modes. A propagating Bessel beam is generated above the radiating waveguide. The Bessel beam is Transverse Magnetic (TM) polarized with a vertical component of electric field that is a zeroth-order Bessel function of the first kind. A higher-order leaky-wave mode is used to reduce losses at millimeter waves and, at the same time, avoid the thin dielectric layers used in previously proposed lower-order leaky-wave Bessel launchers. Closed-form design equations are provided for the proposed structure. In addition, the operating bandwidth of the launcher is defined using dispersion analysis. Near-field measurements of a prototype operating in the frequency range $38-39.5 \mathbf{G H z}$ validate the concept. The measured launcher generates a Bessel beam with a stable spot size of about $4.3 \mathbf{~ m m}(0.57 \lambda)$ over a non-diffractive range of about $16.4 \mathbf{~ m m}$ $(2.2 \lambda)$, within about a $\mathbf{4 \%}$ fractional bandwidth.
\end{abstract}

Index Terms-Bessel beams, near-field focusing, artificial surface, leaky waves, high-order modes, millimeter waves.

\section{INTRODUCTION}

$\mathbf{T}$ HE non-diffractive behavior and focusing/collimating capabilities of Bessel beams make them attractive for a wide range of applications at millimeter waves. In particular, the non-diffractive property may open new possibilities in medical and security imaging, optical manipulation, confinedbeam spectroscopy, etc. [1]-[7]. In brief, ideal Bessel beams are monochromatic localized solutions [8], [9] to the scalar wave equation that do not undergo diffractive spreading when propagating in free space [10]. An infinite radiating aperture is required for the generation of ideal Bessel beams. In practice, it is possible to generate Bessel beams using finite-size radiating aperture at the expense of a limited non-diffractive range. A rigorous vector analysis of the generation of Bessel beams by finite apertures can be found in [5], and [11]-[13], which avoids the paraxial approximation of earlier works [10], [14], [15].

Manuscript received May xx, 2015

W. Fuscaldo is with the Department of Information Engineering, Electronics and Telecommunications, Sapienza University of Rome, 00184 Rome, Italy and with the Institut d'Électronique et de Télécommunications de Rennes (IETR), UMR CNRS 6164, Université de Rennes 1, 35042 Rennes, France (email: fuscaldo@diet.uniroma1.it)

G. Valerio is with the Laboratoire d'Électronique et Électromagnétisme, Université Pierre et Marie Curie, Sorbonnes Universités, 75005 Paris, France.

A. Galli is with the Department of Information Engineering, Electronics and Telecommunications, Sapienza University of Rome, 00184 Rome, Italy.

R. Sauleau and M. Ettorre are with the Institut d'Électronique et de Télécommunications de Rennes (IETR), UMR CNRS 6164, Université de Rennes 1, 35042 Rennes, France.

A. Grbic is with the Radiation Laboratory, Department of Electrical Engineering and Computer Science, and the Center of Photonic and Multiscale Materials (C-PHOM), University of Michigan, Ann Arbor, MI 48109-2122 USA.
A vector analysis of a Bessel-beam launcher working at around $10 \mathrm{GHz}$ is reported in [16] and [17]. It consists of a leaky-wave radial waveguide loaded by an impedance sheet, shown in Fig. 1. In these papers, the lowest-order azimuthallyinvariant TM (with respect to $z$ axis) leaky mode is excited centrally by a coaxial probe. A metallic rim at the edge of the structure is used to reflect an outward going cylindrical wave launched by the feed in order to generate a radiated vertical electric field $E_{z}$ with a $J_{0}$ profile, and a radial field $E_{\rho}$ with a $J_{1}$ profile (zeroth- and first-order Bessel function of the first kind, respectively). Simple design rules are provided, based on the required Bessel profile.

The single mode operation of the launcher in [16] and [17] restricts the separation between the impedance sheet and ground plane of the radial waveguide to thicknesses much smaller than the operating wavelength. Scaling the structure to the millimeter-wave range would require extremely thin waveguides (e.g., a thickness of the dielectric substrate equal to $37.6 \mu \mathrm{m}$ at $f=40 \mathrm{GHz}$ ) leading to restrictively high ohmic losses [18] and practical mechanical problems.

For this reason, here we propose a Bessel-beam launcher at millimeter waves using higher-order TM leaky modes. The structure is again based on a radial waveguide loaded by a capacitive sheet. However, in contrast to the previous designs [16], [17], larger separation distances (on the order of half the wavelength) are considered between the ground plane and the impedance sheet. The launcher can then support higher-order leaky modes and possibly surface waves. A completely novel design approach is thus proposed based on dispersion analysis. Suitable closed-form equations are derived to establish the annular spectrum needed for the generation of propagating

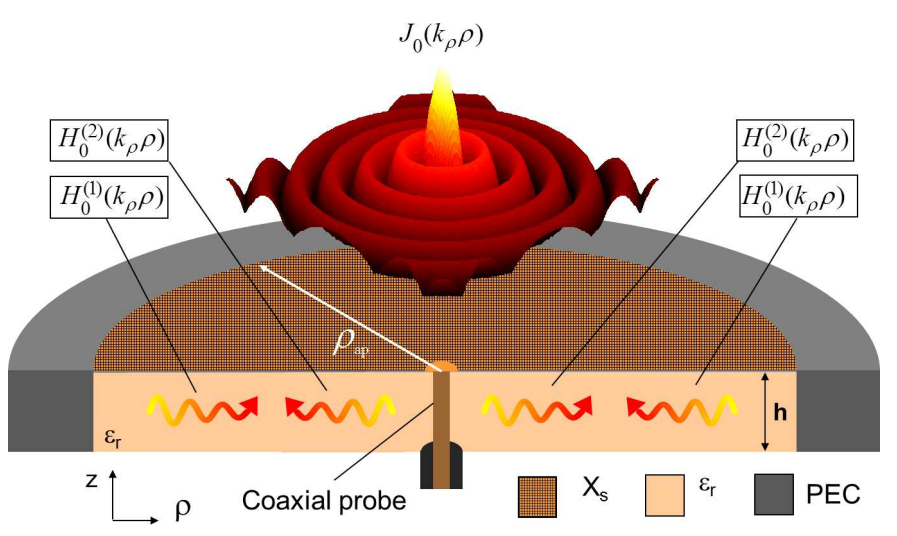

Fig. 1. Illustration of the leaky-mode Bessel-beam launcher under consideration. The red arrows show the outward and inward cylindrical waves launched within the structure by a central coaxial probe. The interference of these cylindrical waves creates a Bessel beam. 
Bessel beams.

The use of higher-order modes requires a frequency range where the presence of lower-order modes and possible surface waves does not affect the pattern of the generated Bessel beam. A bandwidth of operation of the proposed structure is then derived and verified.

The proposed concept and design relations are experimentally validated with a prototype operating at $38.3 \mathrm{GHz}$, which is fabricated using a standard Printed Circuit Board (PCB) fabrication process. Measurements of the vertical electric field demonstrate that, within the considered band $(38-39.5 \mathrm{GHz})$, the generated Bessel beam presents a stable spot size. These results may pave the way for the generation of other kinds of localized waves and in particular X-waves that are a combination of propagating Bessel beams in a defined frequency range [8], [9].

The paper is organized as follows. In Section II, the leaky radial waveguide is presented. In Section III, a modal analysis is outlined and used to derive design equations for the launcher operating with higher-order modes. The maximum achievable bandwidth of the device is also provided. Section IV describes the design of a higher-order Bessel-beam launcher operating at $38.3 \mathrm{GHz}$. In Section V the prototype is reported, and measurement results are shown that validate the design. Finally, conclusions are drawn in Section VI.

\section{LEAKY RADIAL WAVEGUIDE}

This section briefly summarizes the design principles for the generation of a Bessel beam using a launcher that supports lower-order leaky-wave modes [16]. In particular, we consider a TM propagating Bessel beam whose vertical component $(z$ component in Fig. 1) of the electric field $E_{z}$ assumes a zerothorder Bessel function of the first-kind $J_{0}\left(k_{\rho} \rho\right)$, where $k_{\rho}$ is the transverse propagation constant of the generated beam. As presented in [16], the radiated $E_{z}$ field can be expressed as a superposition of an outward traveling cylindrical wave $\left(A_{2} H_{0}^{(2)}\left(k_{\rho} \rho\right)\right.$, a zeroth-order Hankel function of the second kind) and of an inward wave $\left(A_{1} H_{0}^{(1)}\left(k_{\rho} \rho\right)\right.$, a zeroth-order Hankel function of the first kind), whose amplitude coefficients ( $A_{1}$ and $A_{2}$ ) are stipulated by the boundary conditions and excitation. A Bessel function $J_{0}$ can then be generated when the amplitude coefficients are the same $\left(A_{1}=A_{2}\right)$. In this case, the required Bessel function $J_{0}\left(k_{\rho} \rho\right)$ is generated by placing a circular metallic rim at the radial location $(\rho)$ of one of the zeros of $J_{0}\left(k_{\rho} \rho\right)$. The required equations to establish the condition $A_{1}=A_{2}$ can be found in [16], and are reported here for completeness:

$$
\begin{aligned}
& \beta_{\rho} \rho_{\mathrm{ap}} \simeq m \pi-\pi / 4 \quad m=1,2, \ldots \\
& \alpha_{\rho} \rho_{\mathrm{ap}} \ll 1
\end{aligned}
$$

where $\rho_{\text {ap }}$ is the rim radius, and $\beta_{\rho}$ and $\alpha_{\rho}$ are the real and imaginary part of the radial wavenumber, respectively: $k_{\rho}=$ $\beta_{\rho}-j \alpha_{\rho}$. These conditions require that the outward and inward modes are out of phase at $\rho_{\text {ap }}$ and slowly attenuated.
The rim radius $\rho_{\text {ap }}$ is also related to the non-diffractive range $z_{\text {ndr }}$ of the generated Bessel beam through [10]

$$
z_{\mathrm{ndr}}=\rho_{\mathrm{ap}} \sqrt{\left(\frac{k_{0}}{\beta_{\rho}}\right)^{2}-1},
$$

where $k_{0}$ is the free-space wavenumber. As a result, the nondiffractive range can be tuned by varying the value of the transverse propagation constant $\beta_{\rho}$. Alternatively, if $\beta_{\rho}$ and the frequency are fixed, it is possible to obtain larger nondiffractive distances with larger apertures, provided that (1) and (2) are still satisfied. Note that a larger non-diffractive range may be also achieved at the expenses of a wider spotsize. The null-to-null beamwidth (NNBW) is given by

$$
\mathrm{NNBW}=\frac{2 \chi_{0,1}}{\beta_{\rho}},
$$

where $\chi_{0,1}=2.4048$ is the first null of the $J_{0}$ function. Hence, a trade-off exists between the beam size and the maximum non-diffractive range, for a fixed launcher radius.

\section{DeSIGN Of THE STRUCTURE}

In order to investigate the modal properties of the waves supported by the structure in Fig. 1, we solve the dispersion equation of the radial waveguide for TM modes [16], [19]. In the general case of a dielectric-filled leaky radial waveguide the dispersion equation can be expressed by standard transverse resonance technique [20] as

$$
Y_{\text {air }}+Y_{s}-j Y_{0} \varepsilon_{\mathrm{r}} \cot \left(k_{z 1} h\right)=0,
$$

where $Y_{0}=\omega \varepsilon_{0} / k_{z 1}, Y_{a i r}=\omega \varepsilon_{0} / k_{z}$ are the characteristic admittances in the slab and free-space (air), respectively and $Y_{s}$ is the sheet admittance [16]; $k_{z 1}=\sqrt{k_{0}^{2} \varepsilon_{r}-k_{\rho}^{2}}$ and $k_{z}=$ $\sqrt{k_{0}^{2}-k_{\rho}^{2}}$ are the vertical wavenumbers in the slab and in the air, respectively, which are generally complex quantities $\left(k_{z}=\beta_{z}-j \alpha_{z}, k_{z 1}=\beta_{z 1}-j \alpha_{z 1}\right)$. In the following, we will use wavenumbers normalized with respect to $k_{0}$ (identified with a hat) for the sake of simplicity.

In order to solve the dispersion equation for $k_{\rho}$, the values of $Y_{s}$ and $h$ are needed. Conversely, these parameters can be found through (5) once the transverse $k_{\rho}$ or the vertical $k_{z}$ propagation constants of the required Bessel beam are fixed, together with the operating frequency $f_{0}$, and the dielectric constant $\varepsilon_{r}$. Note that when the so-calculated values $\left(Y_{s}\right.$ and $h$ ) are used to solve (5), they give rise to the previously fixed $k_{\rho}$ at the operating frequency $f_{0}$.

In Subsection III.A, we develop simple closed forms in order to determine $Y_{s}$ and $h$ as functions of $\varepsilon_{r}, k_{\rho}$, and $f_{0}$ using (5). In Subsection III.B, we replace these values in the dispersion equation to discuss the overall modal properties of the structure, thus gaining physical insight about the possible excitation of Bessel beams. In Subsection III.C, we provide simple closed-form expressions for the determination of the maximal available bandwidth of the structure. 


\section{A. Closed-form expressions for $X_{s}$ and $h$}

By assuming that the argument of the cotangent function is close to a multiple $n=0,1,2, \ldots$ of $\pi$ [21], a first-order approximation of the cotangent function in (5) can be made,

$$
Y_{\text {air }}+Y_{s}-\frac{j Y_{0} \varepsilon_{\mathrm{r}}}{k_{z 1} h-n \pi}=0
$$

For typical values of the dielectric constant and the radial wavenumber, this approximation is justified when $\left|k_{z 1} h\right|$ is close to $n \pi$. Furthermore, assuming that the impedance sheet is purely reactive $Y_{s}=j B_{s}=-j X_{s}^{-1}$ (as required to support a TM leaky mode [22]), the approximate dispersion equation (6) becomes

$$
\frac{1}{\hat{k}_{z}}-j \frac{\eta_{0}}{X_{s}}-\frac{j \varepsilon_{\mathrm{r}}}{\hat{k}_{z 1}}\left(\frac{1}{k_{0} \hat{k}_{z 1} h-n \pi}\right)=0,
$$

where $\eta_{0}=k_{0} / \omega \varepsilon_{0}$ is the free-space characteristic impedance and $n$ is related to the modal vertical integer index. Following the same procedure outlined in [16], we can rearrange the terms in (7) and solve for $X_{s}$,

$$
X_{s}=-\frac{\hat{k}_{z 1}}{\hat{k}_{z} \epsilon_{r}}\left(j X_{s}+\eta_{0} \hat{k}_{z}\right)\left(k_{0} \hat{k}_{z 1} h-n \pi\right) .
$$

After some algebraic manipulations, a quadratic equation in $h$ is obtained,

$$
a_{2} h^{2}+a_{1} h+a_{0}=0
$$

where

$$
\begin{aligned}
a_{2} & =k_{0}^{2}\left[2 \hat{\alpha}_{z 1} \hat{\beta}_{z 1} w_{1}-\left(\hat{\beta}_{z 1}^{2}-\hat{\alpha}_{z 1}^{2}\right) z_{1}\right] \\
a_{1} & =k_{0}\left\{2 \epsilon_{r} \hat{\alpha}_{z 1} \hat{\beta}_{z 1}\left(\hat{\beta}_{z}^{2}+\hat{\alpha}_{z}^{2}\right)\right. \\
& \left.-n \pi\left[2 \hat{\alpha}_{z 1} \hat{\beta}_{z 1} w_{2}+\hat{\alpha}_{z 1} w_{1}-z_{2}\left(\hat{\beta}_{z 1}^{2}-\hat{\alpha}_{z 1}^{2}\right)-z_{1} \hat{\beta}_{z 1}\right]\right\} \\
a_{0} & =-n \pi\left[\epsilon_{r} \hat{\alpha}_{z 1}\left(\hat{\beta}_{z}^{2}+\hat{\alpha}_{z}^{2}\right)+n \pi\left(\hat{\beta}_{z 1} z_{2}-\hat{\alpha}_{z 1} w_{2}\right)\right],
\end{aligned}
$$

with:

$$
\begin{aligned}
w_{1} & =\left(-\hat{\alpha}_{z} \hat{\alpha}_{z 1}^{2}+\hat{\alpha}_{z} \hat{\beta}_{z 1}^{2}-2 \hat{\alpha}_{z 1} \hat{\beta}_{z 1} \hat{\beta}_{z}\right) \\
w_{2} & =\left(\hat{\beta}_{z 1} \hat{\alpha}_{z}-\hat{\beta}_{z} \hat{\alpha}_{z 1}\right) \\
z_{1} & =\left(\hat{\beta}_{z} \hat{\beta}_{z 1}^{2}-\hat{\beta}_{z} \hat{\alpha}_{z 1}^{2}+2 \hat{\alpha}_{z 1} \hat{\alpha}_{z} \hat{\beta}_{z 1}\right) \\
z_{2} & =\left(\hat{\beta}_{z 1} \hat{\beta}_{z}+\hat{\alpha}_{z 1} \hat{\alpha}_{z}\right) .
\end{aligned}
$$

Finally, by solving (8) and (9) we get:

$$
\begin{aligned}
& h=\frac{-a_{1} \pm \sqrt{a_{1}^{2}-4 a_{2} a_{0}}}{2 a_{2}} \\
& X_{s}=-\frac{\eta_{0} \hat{\alpha}_{z 1}\left(2 k_{0} h \hat{\beta}_{z 1}-n \pi\right)\left(\hat{\beta}_{z}^{2}+\hat{\alpha}_{z}^{2}\right)}{k_{0} h z_{1}-n \pi z_{2}}
\end{aligned}
$$

It is easy to verify that for $n=0$ and $\varepsilon_{\mathrm{r}}=1$ these results coincide with those presented in [16] for the lowest-order leaky-mode launcher. However, in contrast to earlier works [3], [16], higher-order leaky-wave Bessel-beam launchers can generate non-diffractive radiation for two different impedance values (see Eqs. (12) and (13)) and corresponding substrate heights. This gives designers an additional degree of freedom during the design process.

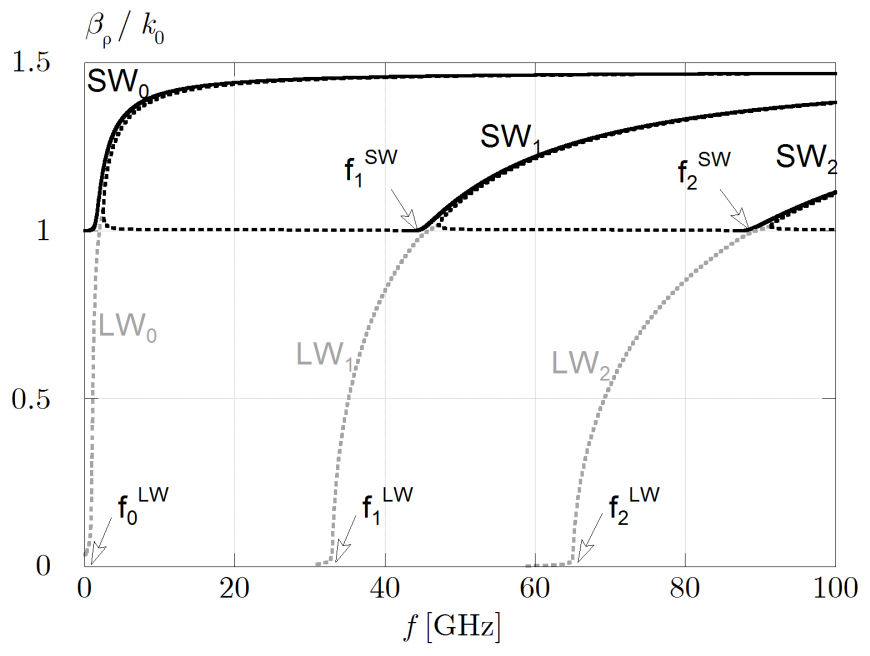

(a)

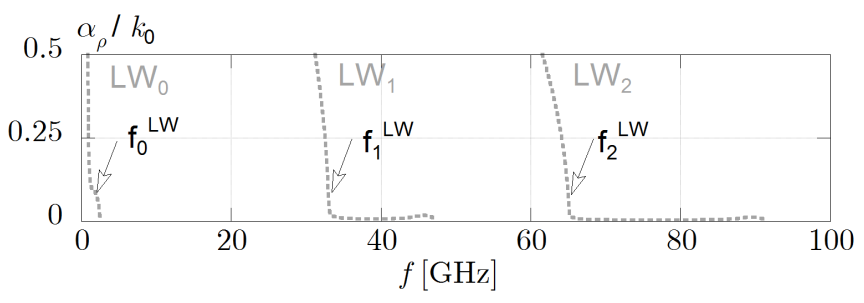

(b)

Fig. 2. (a) Normalized phase constant and (b) normalized attenuation constant vs. frequency $f$ from $\mathrm{DC}$ to $100 \mathrm{GHz}$ for the first TM modes of a partiallyopen layered structure as in Fig. 1. The solid and dashed lines denote the dispersion curves for the proper and improper modes, respectively. The black and grey curves represent the dispersion curves for the real and complex modes, respectively. Hence surface-wave (SW) modes are shown by black solid lines, whereas the leaky-wave (LW) modes are shown by grey dashed lines. In these plots, it is assumed that $X_{s}=-25 \Omega, \varepsilon_{\mathrm{r}}=2.17, h=3.175$ $\mathrm{mm}$.

\section{B. Modal analysis}

The modes supported by the partially-open layered structure are computed by numerically solving eq. (5). The values of $X_{s}$ and $h$ are derived using (12), (13) for a tangential wavenumber $k_{\rho}=(0.8-j 0.007) k_{0}$ at $f \simeq 40 \mathrm{GHz}$ and $\varepsilon_{r}=2.17$. This set of parameters does not limit the general discussion presented in this subsection, since the dispersion diagrams of the structure are qualitatively very similar for a large range of parameters. The numerical parameters chosen here were used for the realized prototype described in Sections IV and V.

Figs. 2(a) and 2(b) provide the normalized phase constant $\beta_{\rho} / k_{0}$ and attenuation constant $\alpha_{\rho} / k_{0}$ as functions of the frequency $f$. Three leaky-wave (LW) modes are observed. By setting $k_{\rho}=0$ in (5), an approximate expression can be obtained for the cutoff frequencies

$$
f_{n}^{\mathrm{LW}} \simeq \frac{c}{2 \pi h \sqrt{\varepsilon_{\mathrm{r}}}} \cot ^{-1}\left(\frac{-\eta_{0}}{X_{s} \epsilon_{r}}\right)+\frac{n c}{2 h \sqrt{\varepsilon_{\mathrm{r}}}}
$$

where $c$ is the light velocity in vacuum. The phase constant of each leaky-wave mode above cutoff varies in the range approximately from 0 to $k_{0}$. As is typical [19], [22]-[25], slightly after $\beta_{\rho}>k_{0}$, the two conjugate leaky poles merge, 
and afterwards give rise to a couple of purely real improper (nonphysical) poles. Specifically, one real improper pole (with a $\beta_{\rho}$ greater than $k_{0}$ ) has a decreasing $\beta_{\rho}$ with respect to the frequency, until it reaches the value $\beta_{\rho}=k_{0}$. At this point, the real improper mode becomes a real proper pole, i.e. a physical surface wave guided at the interface, whose $\beta_{\rho}$ tends to the asymptotic value of $k_{0} \sqrt{\varepsilon_{\mathrm{r}}}$ [19], [22]-[25]. The cutoff frequency of this surface-wave (SW) mode can be determined by replacing $\beta_{\rho}$ with $k_{0}$ in the dispersive equation. Note that a zeroth-order surface wave propagates from DC frequency, since

$$
f_{n}^{\mathrm{SW}} \simeq \frac{n c}{2 h \sqrt{\varepsilon_{\mathrm{r}}-1}}
$$

Since small values of $\alpha_{\rho}$ are always required to launch a Bessel profile with the proposed leaky radial waveguide [16], each leaky-wave mode can be used only at frequencies $f>f_{n}^{L W}$. As is known [26], slow improper waves do not actually contribute to the aperture field, but conversely the excitation of proper surface waves can deteriorate the profile of the generated Bessel beam. It is then important to define the frequency band where a mono-modal leaky-wave propagation can be obtained.

\section{Determination of the maximal available bandwidth}

As was noted in Section II, in order to create a Bessel beam, a circular metallic rim is placed at a radial distance $\rho_{\text {ap }}$ corresponding to a null of the required zeroth-order Bessel function, i.e.

$$
\frac{\beta_{\rho}}{k_{0}}=\frac{\chi_{0, q}}{\rho_{\text {ap }} k_{0}} \quad q=1,2, . .
$$

where $\chi_{0, q}$ is the $q$-th zero of $J_{0}$. The above relation is represented by hyperbolic curves (dashed line in Fig. 3). The intersections with the first higher-order $(n=1)$ leaky-wave $\left(\mathrm{LW}_{1}\right)$ and zeroth-order $(n=0)$ surface-wave $\left(\mathrm{SW}_{0}\right)$ phase constants (see also Fig. 2(a)) identify a grid of resonant modes of the radially waveguide with a metallic rim, as shown in Fig. 3.

The intersection of these curves with the required leakywave mode (here $n=1$ ) defines the operating frequency $f_{0}$ of the Bessel-beam launcher, and the operating phase constant $\hat{\beta}_{0}=\hat{\beta}\left(f_{0}\right)$. In addition, we can define the maximum available bandwidth $B_{\mathrm{av}}$ for mono-modal operation as the smallest frequency range containing $f_{0}$ and delimited at its ends by adjacent intersections with the adjacent resonant modes. It should be stressed that this maximum theoretical bandwidth should not be confused with the practical bandwidth of the launcher, which will likely be narrower and limited by the input impedance match and the frequency dependence of the artificial surface.

In Fig. 3, it is clear that the lower bound of $B_{\mathrm{av}}$ is given by $f_{\text {low }}=\max \left\{f_{\text {inf }}^{\mathrm{SW} 0}, f_{\mathrm{inf}}^{\mathrm{LW} 1}\right\}$, whereas the upper bound is given by $f_{\text {high }}=\min \left\{f_{\text {sup }}^{\mathrm{SW} 0}, f_{\text {sup }}^{\mathrm{LW} 1}\right\}$. These four frequencies can be expressed through simple closed-form relationships. In fact by exploiting the asymptotical periodicity of the zeros of the Bessel functions [27], i.e.

$$
\chi_{0, q+1}-\chi_{0, q} \simeq \pi
$$

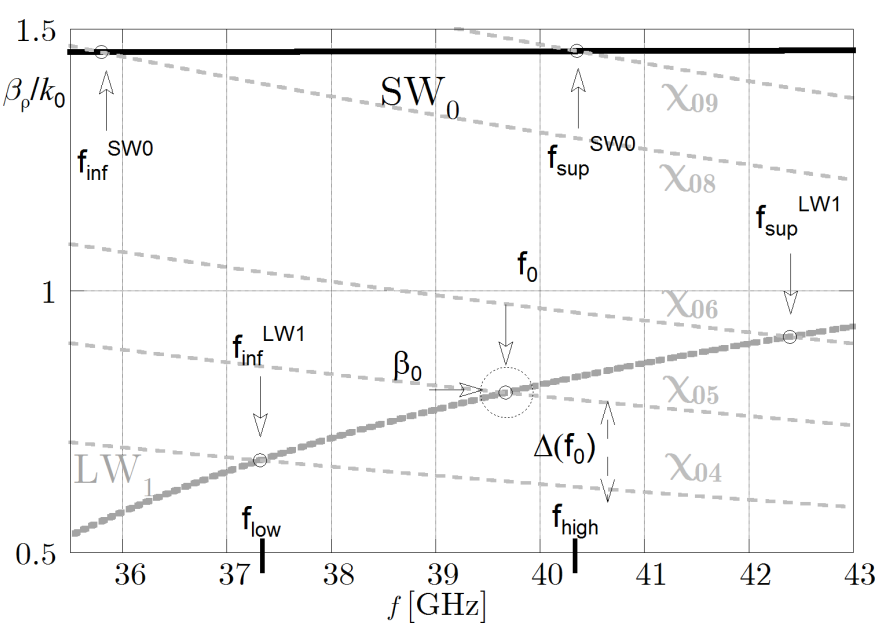

Fig. 3. Dispersion curves for the design of the higher-order launcher prototype. The operating points are given by the intersections of the fast leaky-wave modes and the hyperbolic curves given by the Bessel zeros. Once the operating point is chosen, the operating bandwidth is fixed by the closest intersections of either the fast leaky-wave or the surface-wave modes. The parameters used in Fig. 2 are also assumed here.

from (16) the vertical distance $\Delta$ between two consecutive hyperbolas at a given frequency $f$ is given by

$$
\Delta(f)=\frac{\chi_{0, q+1}}{\rho_{\text {ap }} k_{0}}-\frac{\chi_{0, q}}{\rho_{\text {ap }} k_{0}} \simeq \frac{c_{0}}{2 \rho_{\text {ap }} f} .
$$

By assuming a nearly linear leaky-wave phase constant between $f_{\text {inf }}^{\mathrm{LW} 1}$ and $f_{\text {sup }}^{\mathrm{LW} 1}$, we get

$$
f_{\mathrm{inf}, \sup }^{\mathrm{LW} 1}=f_{0} \pm \frac{\Delta\left(f_{0}\right)}{\xi+\psi},
$$

where

$$
\xi=\left.\frac{d \hat{\beta}_{\rho}^{\mathrm{LW} 1}(f)}{d f}\right|_{f_{0}}, \quad \psi=\frac{\chi_{0, q} c_{0}}{2 \pi \rho_{\mathrm{ap}} f_{0}^{2}} .
$$

Moreover, if the fundamental surface-wave phase constant is close to the asymptotic value $\hat{\beta}_{\rho}^{\text {SW0 }} \simeq \sqrt{\varepsilon_{\mathrm{r}}}$, we have

$$
f_{\mathrm{inf}}^{\mathrm{SW0}}=\frac{\chi_{0, r} c_{0}}{2 \pi \rho_{\mathrm{ap}}}, f_{\mathrm{sup}}^{\mathrm{SW} 0}=\frac{\chi_{0, r+1} c_{0}}{2 \pi \rho_{\mathrm{ap}}}
$$

where

$$
r=\left\lfloor\frac{\rho_{\mathrm{ap}} k_{0} \delta \beta^{\mathrm{SW} 0}\left(f_{0}\right)}{\pi}\right\rfloor,
$$

$\delta \beta^{\mathrm{SW0}}\left(f_{0}\right)=\sqrt{\varepsilon_{\mathrm{r}}}-\hat{\beta}_{0}$ being the phase difference between the leaky wave and the fundamental surface wave at the operating frequency $f_{0}$, and $\lfloor\cdot\rfloor$ is the floor function.

\section{NUMERICAL VALIDATION}

\section{A. Design procedure for the Bessel-beam launcher}

A leaky radial waveguide exciting a Bessel beam with $k_{\rho}=$ $(0.8-j 0.007) k_{0}$ around the operating frequency of $f_{0}=39.7$ $\mathrm{GHz}$ has been designed to validate the results of the previous section. The value of $\rho_{\text {ap }}$ is fixed by $f_{0}$, whereas $h, X_{s}$ are derived using (12) and (13) once the radial $(q)$ and vertical $(n)$ order of the resonance are chosen. A choice of $q=5$, the fifth 


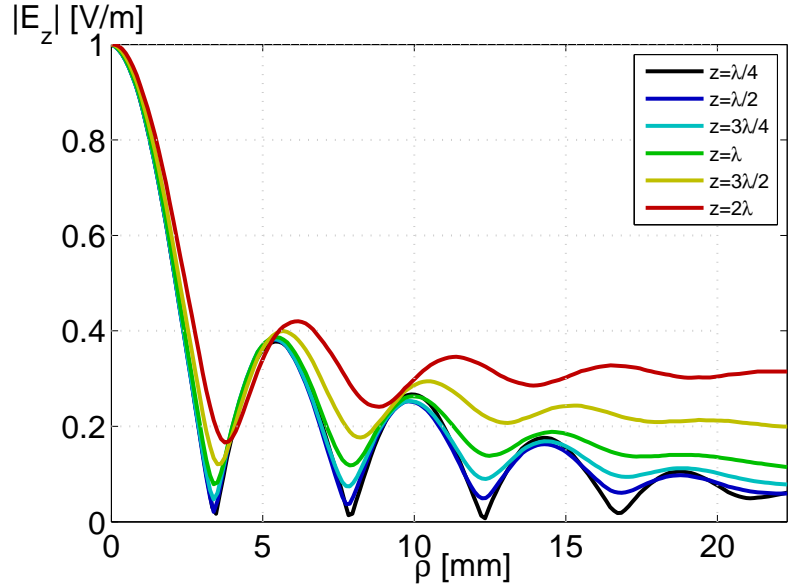

Fig. 4. Radial behavior of the normalized electric field $\left|E_{z}\right|$ at $f=39.7$ $\mathrm{GHz}$, for the launcher under analysis (parameters as in Fig. 2), for various distances from the aperture, computed using COMSOL Multiphysics.

zero of the Bessel function, and $n=1$, the first higher-order leaky-wave mode, leads to the following set of parameters:

$$
\begin{aligned}
& \rho_{\text {ap }}=22.3 \mathrm{~mm}, \\
& h=3.175 \mathrm{~mm}, \\
& X_{s}=-25 \Omega
\end{aligned}
$$

where we have considered a dielectric substrate with a relative permittivity $\varepsilon_{\mathrm{r}}=2.17$ as in Section III. These values lead to the desired operating point with a relative accuracy on the order of $1 \%$, in agreement with the small-argument firstorder approximation of the cotangent function made in Subsection III-A. The dispersion diagram of the structure in the range of interest is shown in Fig. 3. Note that a mono-modal propagation is obtained between frequencies $f_{\text {low }}=37.3 \mathrm{GHz}$ and $f_{\text {high }}=40.3 \mathrm{GHz}$, respectively.

The operation of the structure as a Bessel-beam launcher has been validated numerically using COMSOL Multiphysics [28]. The artificial surface is modeled as an infinitesimally thin layer. In Fig. 4, the computed normalized vertical electric field $E_{z}(\rho)$ at different distances $(z)$ from the radiating aperture is shown. Clearly, a Bessel beam is generated at the expected frequency $f=39.7 \mathrm{GHz}$. The typical Bessel function $J_{0}$ profile is observed up to the non-diffractive range, equal to $16.4 \mathrm{~mm} \simeq 2.2 \lambda_{0}\left(\lambda_{0}=7.5 \mathrm{~mm}\right.$, at $\left.f_{0}=40 \mathrm{GHz}\right)$, where the sidelobes are no longer clearly distinct, and the main beam starts to widen. This behavior is evident in Fig. 5(a), which shows the same electric field component in a 2-D plot along the $\rho z$-plane. The full-wave simulation confirms the operation of the radial waveguide as a Bessel-beam launcher.

\section{B. Bandwidth verification}

In this subsection, 2-D plots of $E_{z}$ along the $\rho z$-plane are shown at both the lower $f_{\text {low }}$ and higher $f_{\text {high }}$ limits of the maximum available bandwidth, in order to validate the closedform expressions (19) and (21) for the bandwidth. At the lower limit $f_{\text {low }}=37.3 \mathrm{GHz}$, the radiated $E_{z}$ field has a larger beamwidth (see Fig. 5(b)). This is due to the smaller transverse propagation constant of the leaky-wave mode which intersects the hyperbolic curve corresponding to $q=4$. At the upper end, $f_{\text {high }}=40.3 \mathrm{GHz}$, the $E_{z}$ radiated field is spoiled by the excitation of a surface wave, and hence no useful focused beam is generated (see Fig. 5(c)). However, within the maximum available bandwidth $37.3-40.3 \mathrm{GHz}$, a Bessel beam with a quasi-constant spot size can be observed, as confirmed in the following section by experimental tests.

\section{PRototype AND MEASUREMENTS}

\section{A. Realization}

The Bessel launcher designed in the previous section has been fabricated using a PCB process at IETR, Rennes, France. The radial waveguide consists of a substrate with permittivity $\varepsilon_{\mathrm{r}}=2.17$ (Neltec NY9217) and height $h=3.175 \mathrm{~mm}$. The sheet reactive impedance $\left(X_{s} \simeq-25 \Omega\right)$ is realized by etching a two-dimensional array of interleaved squared metallic patches [17] (see Fig. 6) with a periodicity $p=750 \mu \mathrm{m}$ $\left(\lambda_{0} / 10\right.$ at $\left.f_{0}=40 \mathrm{GHz}\right)$ and a border distance $d=50 \mu \mathrm{m}$ on a double-side patterned substrate of permittivity $\varepsilon_{\mathrm{r}}=6.15$ (Rogers Duroid 6006) and height $h_{\mathrm{FSS}}=0.127 \mathrm{~mm}$. Fullwave simulations with Ansys HFSS [29] have been used to design the impedance sheet [30]. The impedance sheet has been glued to the radial waveguide using an adhesive layer Taconic fastRise FR-27-0030-25 of permittivity $\varepsilon_{\mathrm{r}}=2.78$

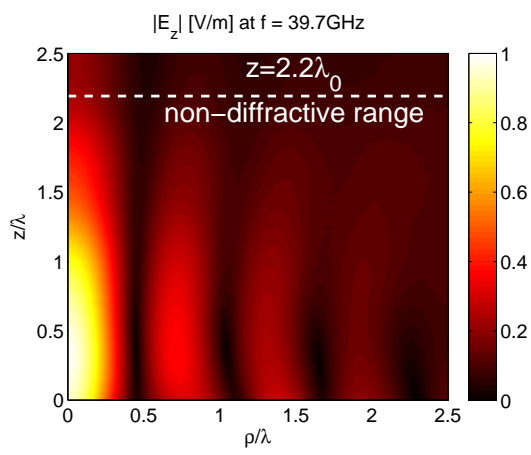

(a)

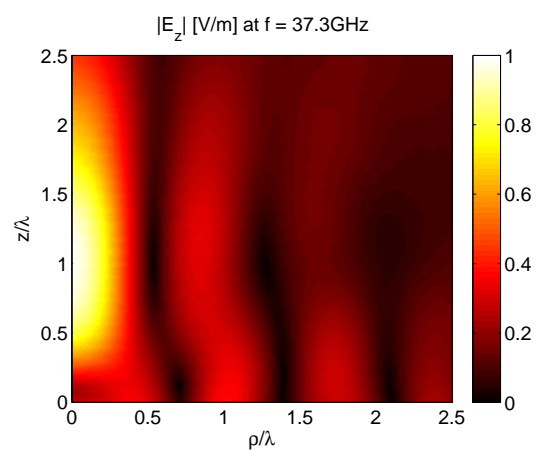

(b)

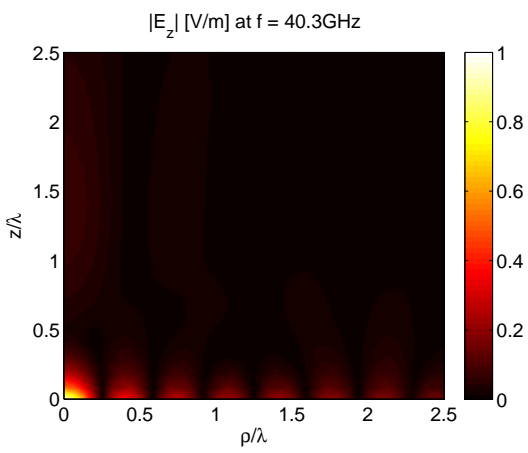

(c)

Fig. 5. Contour plot of the electric field $\left|E_{z}\right|$ along the $\rho z$-plane for the mm-wave launcher under analysis at (a) $f=39.7 \mathrm{GHz}$, (b) $f=37.3 \mathrm{GHz}$, and (c) $f=40.3 \mathrm{GHz}$. 
TABLE I

GEOMETRICAL DIMENSIONS FOR THE MATCHING NETWORK OF THE MM-WAVE BESSEL-BEAM LAUNCHER

\begin{tabular}{c|c}
\hline Parameter & Value [mm] \\
\hline \hline & \\
\hline$r_{\text {disk1 }}$ & 0.65 \\
$r_{\text {disk2 }}$ & 0.69 \\
$r_{\text {match }}$ & 0.20 \\
$r_{\text {in }}$ & 0.19 \\
$h_{\text {FSS }}$ & 0.13 \\
\hline
\end{tabular}

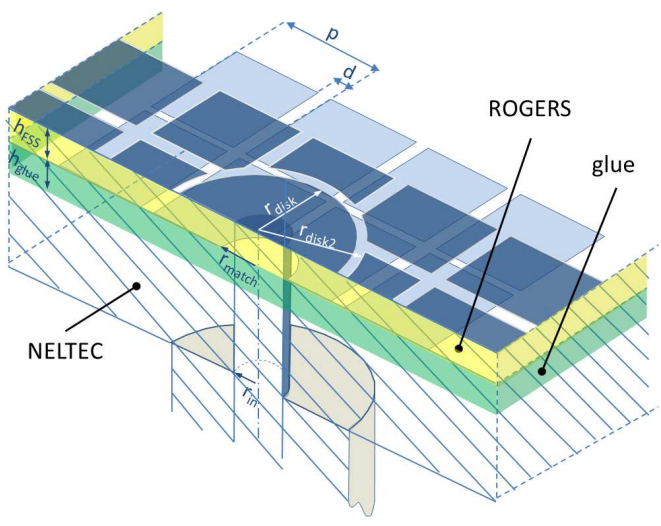

Fig. 6. Schematic of the coaxial probe transition used for matching the mmwave launcher.

and thickness $h_{\text {glue }}=86 \mu \mathrm{m}$. A commercial connector (SRI Connector Gage 85131100080) has been used to feed the structure from the back side of the launcher. For matching purposes, the geometry of the impedance surface has been modified close to the inner probe of the coaxial connector, as shown in detail in Fig. 6. In fact the radius of the metallic disk $r_{\text {disk1 }}$ and its distance from the adjacent patches are critical parameters for impedance matching. The final design parameters are reported in Table I. It should be noted that the inner probe of the coax is only connected to the upper layer of the impedance sheet. The stacked structure is placed within a circular waveguide of radius $\rho_{\text {ap }}$. The final prototype is shown

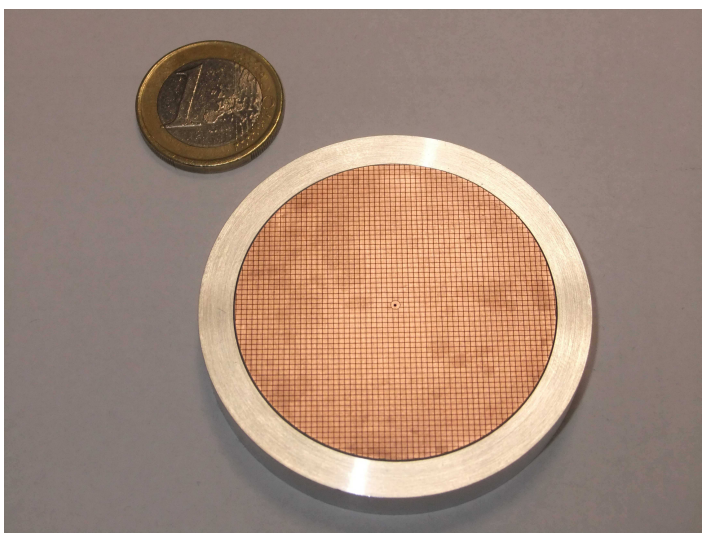

Fig. 7. Prototype of the mm-wave leaky-mode Bessel-beam launcher. The feeding probe can be recognized at the center of the structure.

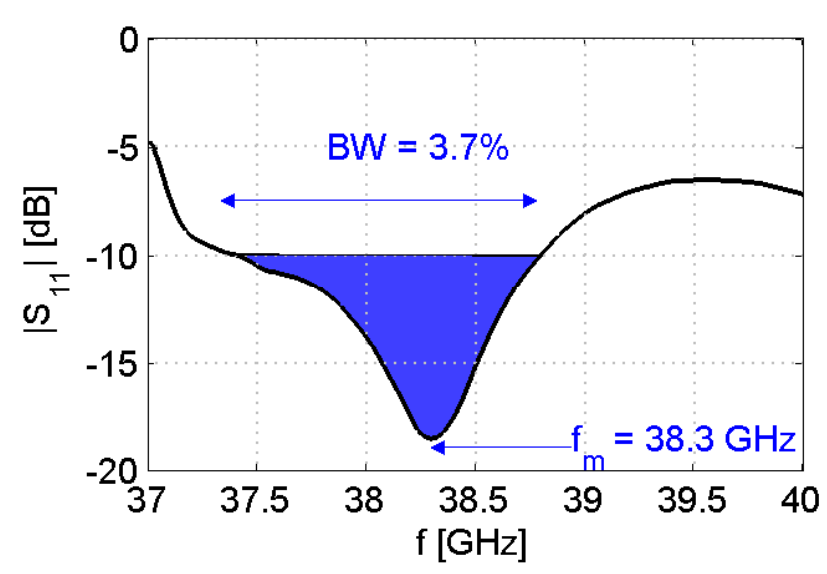

(a)

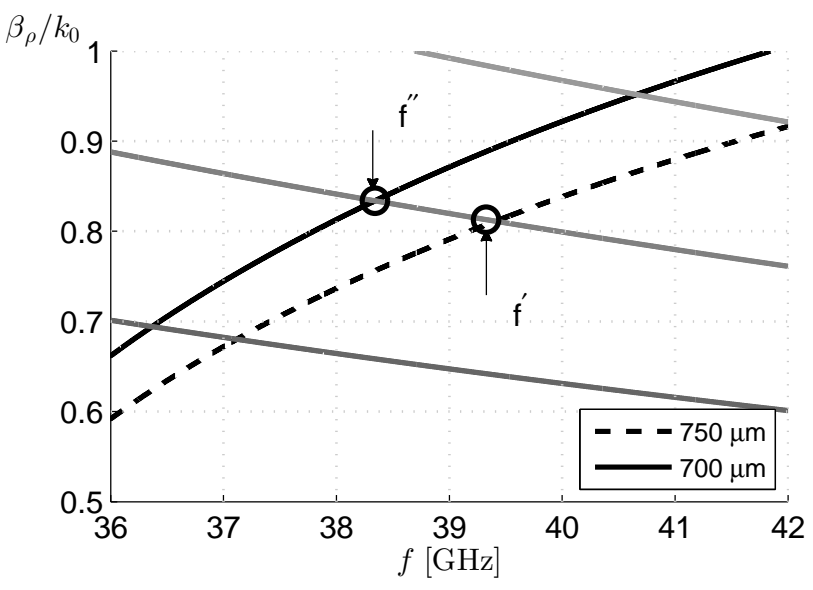

(b)

Fig. 8. (a) Measured reflection coefficient ( $\left|S_{11}\right|$ in $\left.\mathrm{dB}\right)$ of the prototype in the frequency range $37 \div 40 \mathrm{GHz}$. The blue region highlights the frequency range for which the return loss is under $-10 \mathrm{~dB}$. (b) Effects of fabrication tolerances for the launcher under analysis. Dispersion curves within the frequency range $36 \div 42 \mathrm{GHz}$. The dashed and solid lines denote the dispersion curves for the realized impedance sheet with a patched period of $p=750 \mu \mathrm{m}$ and $p=700 \mu \mathrm{m}$, respectively. The distance $d$ is equal to $50 \mu \mathrm{m}$, in both cases. A shift of the operating point from $f=39.7 \mathrm{GHz}$ down to $f^{\prime \prime}=38.3 \mathrm{GHz}$ is observed.

in Fig. 7. The prototype has been measured in the frequency range $38-39.5 \mathrm{GHz}$ with a frequency step of $100 \mathrm{MHz}$, and compared with full-wave results of the complete structure performed in HFSS. The vertical component of the electric field $E_{z}$ has been measured above the prototype, using a small electric probe scanning an area of $45 \mathrm{~mm} \times 45 \mathrm{~mm}$ with a step of $0.75 \mathrm{~mm}$ at six different $z$-planes starting from $3.75 \mathrm{~mm}\left(\lambda_{0} / 2\right)$ up to $18 \mathrm{~mm}\left(5 \lambda_{0} / 2\right)$, in order to verify the Bessel beam generation. This probe is made of a semirigid coaxial cable (UT-85) with the inner conductor extending $0.75 \mathrm{~mm}\left(\lambda_{0} / 10\right.$ at $\left.f_{0}=40 \mathrm{GHz}\right)$ beyond the outer conductor and dielectric. In addition, the $E_{z}$ component has been also measured along the $x z$-plane $\left(\phi=0^{\circ}\right)$ in order to highlight the overall non-diffractive behavior of the generated Bessel beam within the expected non-diffractive range.

Through measurements, a minimum in reflection coefficient of the prototype was found at a frequency of $f_{m}=38.3 \mathrm{GHz}$ 


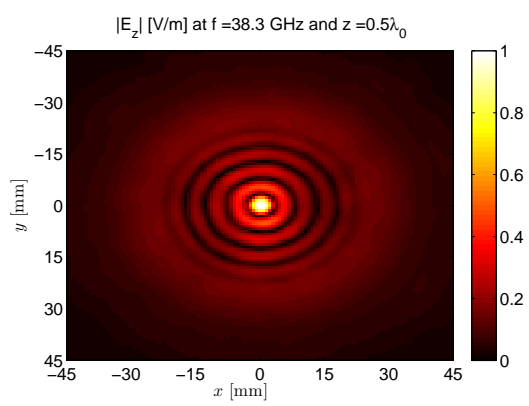

(a)

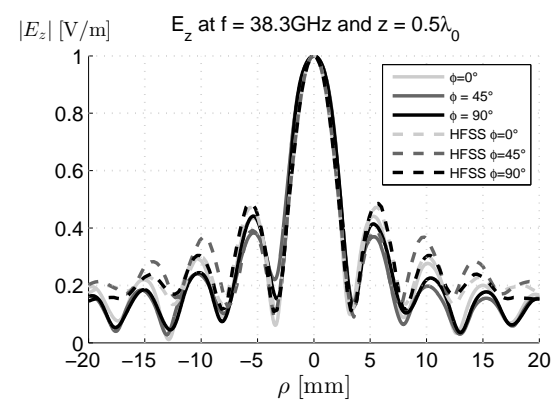

(d)

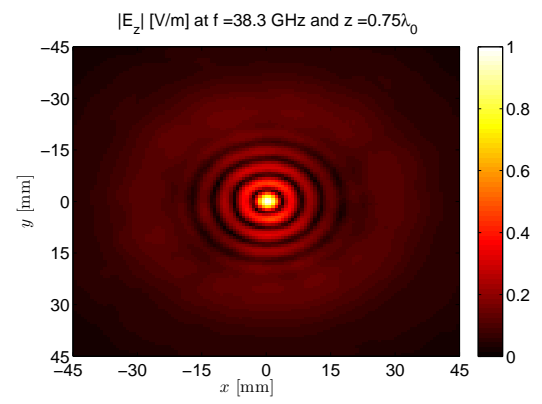

(b)

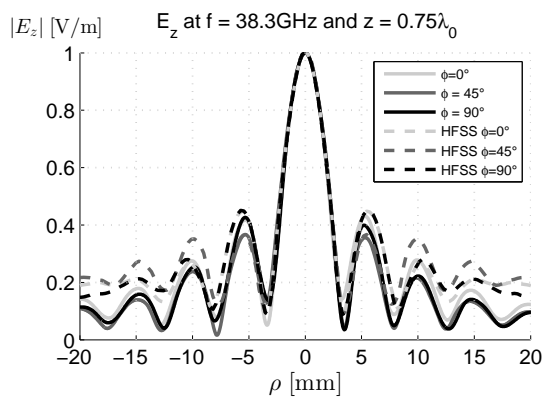

(e)

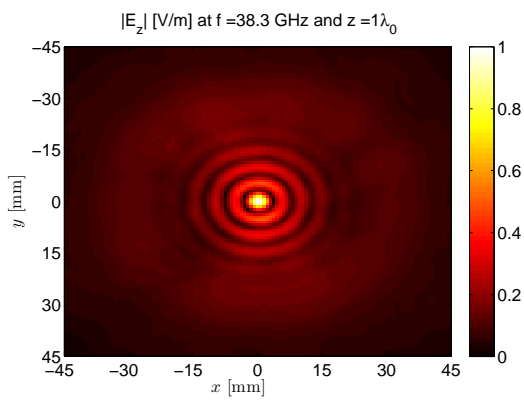

(c)

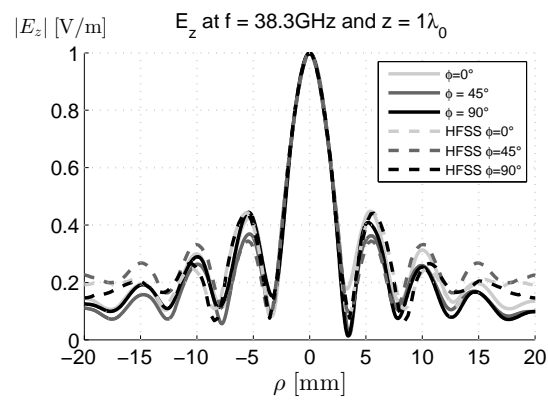

(f)

Fig. 9. Measured patterns of the normalized $E_{z}$ component of the electric field at $f=38.3 \mathrm{GHz}$ at three different distances $z=0.5 \lambda_{0}, 0.75 \lambda_{0}, 1 \lambda_{0}$ from the impedance surface, where $\lambda_{0}$ equal to $7.5 \mathrm{~mm}$. (a)-(c) Contour plots of the measured field. (d)-(f) A comparison between measurements and simulations along different azimuthal $\phi$ planes.

as shown in Fig. 8(a). Around this frequency, the measured $\left|S_{11}\right|$ shows a $-10 \mathrm{~dB}$ fractional bandwidth of approximately $3.7 \%$. It is noted that $f_{m}$ is shifted of $2.5 \%$ relative to the expected operating frequency $f_{0}$. This frequency shift may be attributed to fabrication tolerances. Indeed, by assuming a systematic error of $50 \mu \mathrm{m}$ (a value that fits the PCB industry standard tolerances) for the realization of the periodic impedance sheet in the dispersion analysis of the prototype, as shown in Fig. 8(b), an operating point $f^{\prime \prime}$ equal to $38.3 \mathrm{GHz}$ is found (see solid line in Fig. 8(b)), in agreement with the $2.5 \%$ shift observed in measurements. Therefore, in the following subsections we provide measurements results within the measured frequency range $38-39.5 \mathrm{GHz}$, with an operating frequency equal to $f_{m}=38.3 \mathrm{GHz}$. It is worth mentioning that fabrication tolerances also affect the available bandwidth (refer to Subsection III-C) by shifting downward the lower limit of the bandwidth to $f=36.4 \mathrm{GHz}$. On the other hand, the upper limit remains unaffected.

\section{B. Bessel beam profile}

Contour plots of the measured $E_{z}$ component of the electric field above the aperture are shown in Figs. 9(a)-(c) at various distances $\left(z=0.5 \lambda_{0}, 0.75 \lambda_{0}, 1.0 \lambda_{0}\right.$, where $\lambda_{0}$ is equal to $7.5 \mathrm{~mm}$ ) from the radiating aperture at the operating frequency $38.3 \mathrm{GHz}$. An azimuthally symmetric Bessel beam is clearly shown. In particular, it is possible to distinguish the five dark rings corresponding to the five nulls of the Bessel function.

Figs. 9(d)-(f) provide detailed measurements of the normalized $E_{z}$ component along various azimuthal planes at fixed distances from the radiating aperture. Full-wave results
(HFSS) are also provided for comparison. Measurements and simulations are in very close agreement, thereby validating the proposed analysis and design procedures.

Finally, Fig. 10 provides the measured $E_{z}$ field component along the $x z$-plane. As expected, the spot size represented here by the Half-Power Beamwidth (HPBW) and the Bessel profile are preserved along the axis of propagation up to the non-diffractive range $z=16.4 \mathrm{~mm}$ (marked with a white line). Above this distance, diffraction prevails, and the beam starts to widen.

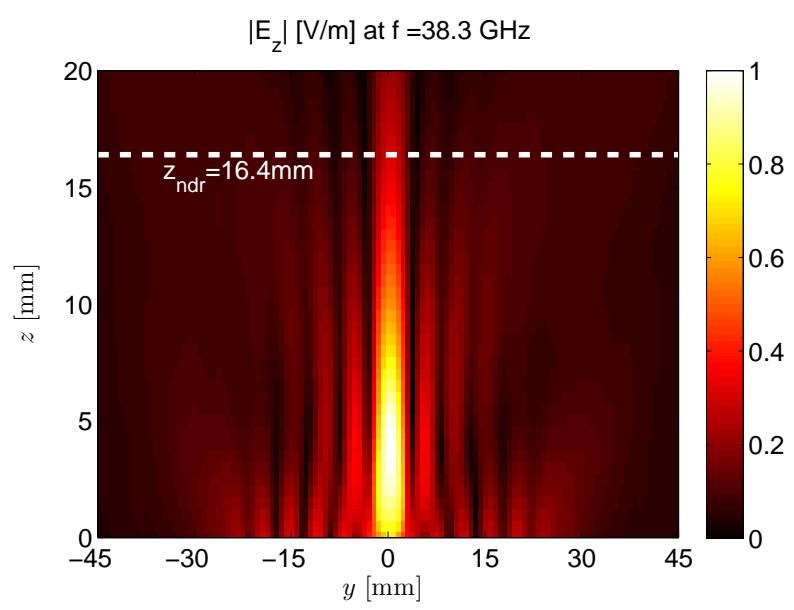

Fig. 10. Contour plots of the measured $E_{z}$ component of the normalized electric field at $f=38.3 \mathrm{GHz}$ along the $x z$-plane. The figure confirms the limited-diffraction behavior of the launcher. 


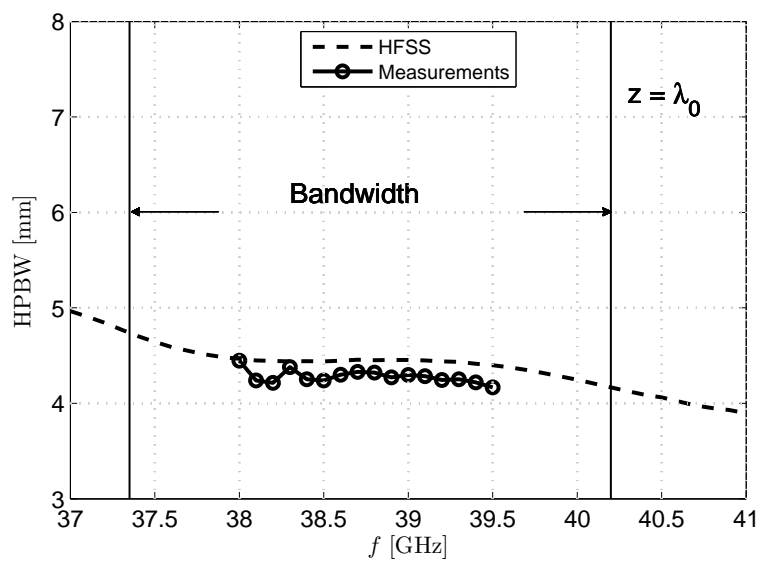

Fig. 11. Variation of the HPBW with frequency for the mm-wave launcher prototype. Full-wave simulation results are shown with dashed lines from 37 to $41 \mathrm{GHz}$. Measurements are shown with circles, from $38 \mathrm{GHz}$ to $39.5 \mathrm{GHz}$. The vertical lines mark the extrema of the maximum available bandwidth, as predicted by (19) and (21).

\section{Beamwidth variation versus frequency}

In this section, the variation of the measured HPBW with the frequency at a fixed distance $\left(z=\lambda_{0}\right)$ from the radiating aperture is reported, and compared to full-wave simulation results (HFSS). As predicted by (4), due to the dispersive behavior of $\beta_{\rho}$, both the simulated (see dashed line in Fig. 11) and the measured HPBW (see circles in Fig. 11) decrease monotonically slowly with frequency, within the maximum available bandwidth (37.3-40.3 GHz) given by (19) and (21). Moreover, the measured HPBW remains quasi-stable over the restricted frequency range $38-39.5 \mathrm{GHz}$ with a variation coefficient $(\mathrm{CV})$ :

$$
\mathrm{CV}=\frac{\sigma_{\mathrm{HPBW}}}{\overline{\mathrm{HPBW}}} \simeq 1.1 \%
$$

being $\sigma_{\mathrm{HPBW}} \simeq 50 \mu \mathrm{m}$ the standard deviation of the measured $\mathrm{HPBW}$, and $\overline{\mathrm{HPBW}} \simeq 4.3 \mathrm{~mm}$ is its mean value.

In contrast to [17], the Bessel beam profile is not only verified along the axis of propagation within the non-diffractive range at the operating frequency, but also over a considerable bandwidth.

\section{CONCLUSION}

We have presented the design and implementation of a leaky radial launcher operating in the millimeter-wave range that supports higher-order leaky modes in order to generate propagating Bessel beams. Higher-order leaky modes allow for suitable designs with PCB fabrication. In particular, the substrate thickness is no longer impractically thin, with consequent reduced losses if compared to lower-order leaky-wave launcher design. The proposed modal analysis has shown that an operating bandwidth exists over which the simultaneous presence of surface waves and dominant (lower-order) leaky modes does not affect the generation of the Bessel beam. Simple closed formulas useful for the design and for the limits of the frequency band have been provided.

Measurement results of a prototype operating around $38.3 \mathrm{GHz}$ have confirmed the proposed analysis and design.
In particular, it has been verified that within the defined bandwidth, the generated Bessel beam maintains a quasiconstant spot size. The possibility of generating zeroth-order Bessel beams with fixed a spot size over a certain bandwidth can also pave the way for the creation of soliton-like solutions, such as X-shaped pulses.

\section{ACKNOWLEDGMENTS}

The authors would like to thank the European Science Foundation (NEWFOCUS project), the Agence National de la Recherche (ANR-14-CE26-0030-01, WIREFREE), and CNRS (PICS project) for their support.

\section{REFERENCES}

[1] R. M. Lapointe, "Review of non-diffracting Bessel beam experiments," Opt. Las. Tech., vol. 24, no. 6, pp. 315-321, Dec. 1992.

[2] T. A. Herman and R. M. Wiggins, "Production and uses of diffractionless beams," J. Opt. Soc. A, vol. 8, no. 6, pp. 932-942, Jun. 1991.

[3] P. Lemaitre-Auger, S. Abielmona, and C. Caloz, "Generation of Bessel beams by two-dimensional antenna arrays using sub-sampled distributions," IEEE Trans. Antennas Propag., vol. 61, no. 4, pp. 1838-1849, Apr. 2014.

[4] A. Mazzinghi, M. Balma, D. Devona, G. Guarnieri, G. Mauriello, and M. Albani, "Large depth of field pseudo-Bessel beam generation with a RLSA antenna," IEEE Trans. Antennas Propag., vol. 62, no. 8, pp. 3911-3919, Aug. 2014.

[5] M. Albani, S. C. Pavone, M. Casaletti, and M. Ettorre, "Generation of non-diffractive Bessel beams by inward cylindrical travelling wave aperture distributions," Opt. Exp., vol. 22, no. 15, pp. 18354-18364, Jul. 2014.

[6] D. McGloin and K. Dholakia, "Bessel beams: diffraction in a new light," Contemporary Physics, vol. 46, no. 1, pp. 15-28, Jan. 2005.

[7] C. Pfeiffer and A. Grbic, "Generating stable tractor beams with dielectric metasurfaces," Phys. Rev. B, vol. 91, no. 11, pp. 115408-115415, Mar. 2015.

[8] H. E. Hernández Figueroa, M. Zamboni-Rached, and E. Recami, "Localized waves: a historical and scientific introduction," in Localized Waves, ed. New York: John Wiley \& Sons, 2008, pp. 7-16.

[9] J. Y. Lu and J. F. Greenleaf, "Nondiffracting X waves - exact solutions to free-space scalar wave equation and their finite aperture realizations," IEEE Trans. Ultrason. Ferroelectr. Freq. Control, vol. 39, no. 1, pp. 19-31, Jan. 1992

[10] J. Durnin, "Exact solutions for nondiffracting beams. I. The scalar theory," J. Opt. Soc. Amer., vol. 4, no. 4, pp. 651-654, Apr. 1987.

[11] S. R. Mishra, "A vector wave analysis of a Bessel beam," Opt. Commun., vol. 85, no. 2-3, pp. 159-161, Sep. 1991.

[12] Z. Bouchal and M. Olivik, "Non-diffractive vector Bessel beams," Journal of Modern Optics, vol. 42, no. 8, pp. 1555-1556, Jan. 1995.

[13] F. G. Mitri, "Three-dimensional vectorial analysis of an electromagnetic non-diffracting high-order Bessel trigonometric beam," Wave Motion, vol. 49, no. 5, pp. 19-31, Jul. 1992.

[14] S. Monk, J. Arlt, D. A. Robertson, J. Courtial, and M. J. Padgett, "The generation of Bessel beams at millimetre-wave frequencies by use of an axicon," Opt. Commun., vol. 170, no. 4 pp. 213-215, Nov. 1999.

[15] J. Arlt and K. Dholakia, "Generation of high-order Bessel beams by use of an axicon," Opt. Commun., vol. 177, no. 4, pp. 297-301, Apr. 2000.

[16] M. Ettorre and A. Grbic, "Generation of propagating Bessel beams using leaky-wave modes," IEEE Trans. Antennas Propag., vol. 60, no. 8, pp. 3605-3613, Aug. 2012.

[17] M. Ettorre, S. M. Rudolph, and A. Grbic, "Generation of propagating Bessel beams using leaky-wave modes: experimental validation," IEEE Trans. Antennas Propag., vol. 60, no. 7, pp. 2645-2653, Jul. 2012.

[18] D. M. Pozar, Microwave Engineering, 2nd ed. New York: John Wiley \& Sons, 1998, ch. 3, sec. 2, pp. 112-118.

[19] G. Valerio, D. R. Jackson, and A. Galli, "Fundamental properties of surface waves in lossless stratified structures," Proc. R. Soc. A, vol. 466, pp. 2447-2469, Mar. 2010.

[20] G. Valerio, D. R. Jackson, and A. Galli, "Formulas for the number of surface waves on layered structures," IEEE Trans. Microw. Theory Tech., vol. 58, no. 7, pp. 1786-1795, Dec. 2010. 
[21] W. Fuscaldo, G. Valerio, A. Galli, R. Sauleau, and M. Ettorre "A millimeter-wave Bessel-beam launcher through the excitation of higherorder leaky modes," 8th Europ. Conf. Antennas Propag. (EuCAP 2014), The Hague, The Netherlands, 2014, pp. 1439-1441.

[22] A. Hessel, "General characteristics of traveling wave antennas," in Antenna Theory, R. E. Collin, and R. F. Zucker, New York: McGrawHill, 1969.

[23] A. A. Oliner and D. R. Jackson, "Leaky Wave Antennas," in J. L. Volakis (ed.) Antenna Engineering Handbook, 4th ed. New York: McGraw-Hill, 2007.

[24] P. Lampariello, F. Frezza, and A. A. Oliner, "The transition region between bound-wave and leaky-wave ranges for a partially dielectricloaded open guiding structure," IEEE Trans. Microw. Theory Tech., vol. 38, no. 12, pp. 1831-1836, Dec. 1990.

[25] C. Di Nallo, F. Frezza, A. Galli, P. Lampariello, and A. A. Oliner, "Properties of NRD-guide and H-guide higher-order modes: physical and nonphysical ranges," IEEE Trans. Microw. Theory Tech., vol. 42, no. 12, pp. 2429-2434, Dec. 1994.

[26] T. Tamir and A. A. Oliner, "Guided complex waves: Part 1. Fields at an interface," Proc. I.E.E., vol. 110, no. 2, pp. 310-324, Feb. 1963.

[27] G. N. Watson, A Treatise on the Theory of Bessel Functions, London: Cambridge University Press, 1966, ch. 15, sec. 52, pp. 503-505.

[28] COMSOL Multiphysics Version 4.2a 1998-2011.

[29] Ansys HFSS Version 16.0 1984-2015, Ansys Corporation.

[30] B. H. Fong, J. S. Colburn, J. J. Ottusch, J. L. Visher, and D. F Sievenpiper, "Scalar and tensor holographic artificial impedance surfaces," IEEE Trans. Antennas Propag., vol. 58, no. 10, pp. 3212-3221, Oct. 2010.

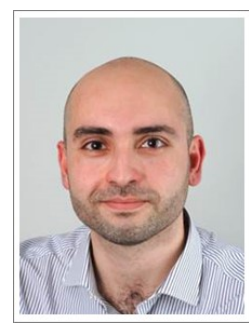

Guido Valerio (S'06-M'10) received the M.S. degree (cum laude and honorable mention) in electronic engineering in 2005, and the Ph.D. degree in electromagnetics in 2009, from La Sapienza University, Rome, Italy. From February to August 2008 he was a Visiting Scholar at the University of Houston, TX, USA. From 2011 to 2014, he was a researcher at the Institute dElectronique et de Telecommunications de Rennes (IETR), France. Since September 2014 he is an Associate Professor in the Electronics and Electromagnetism Laboratory, at Sorbonne Universités, Université Pierre et Marie Curie, in Paris, France. His scientific interests involve numerical methods for wave propagation and scattering in complex structures; namely, efficient computation of periodic Green's functions, the interaction of nonperiodic sources with periodic media, modal properties of multilayered structures, full-wave methods for large multilayered SIW. He has worked in the GPR characterization of Martian soil, and in the design of UWB antennas, harmonic-tunable active antennas, leaky-wave antennas, multifunction antenna arrays. In 2008 Dr. Valerio was the recipient of the "Leopold B. Felsen Award for Excellence in Electrodynamics". In 2009 he was a finalist for the "Young Engineering Prize" at the European Microwave Conference. In 2010 he was the recipient of the "Barzilai Prize" for the best paper at the National Italian Congress of Electromagnetism (XVIII RiNEm). In 2014, he was the recipient of the RMTG Award for junior researchers presented at the IEEE Antennas and Propagation Society Symposium, Memphis, TN.

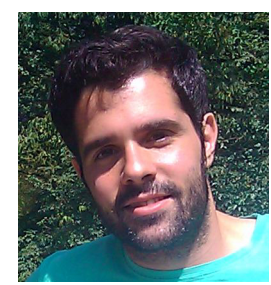

Walter Fuscaldo (S'15) was born in Rome, Italy, in 1987. He received the B.Sc. and M.Sc. (cum laude) degrees in telecommunications engineering from Sapienza University of Rome, Rome, Italy, in 2010 and 2013, respectively. He is currently pursuing the Ph.D. degree in cotutelle agreement between the Department of Information Engineering, Electronics and Telecommunications, Sapienza University of Rome, Rome, Italy and the Institut d'Électronique et de Télécommunications de Rennes (IETR), Université de Rennes 1, France. His research interests include leaky-wave antennas, millimeter-wave focusing systems, terahertz antennas, and graphene metasurfaces. Mr. Fuscaldo was the recipient of the Yarman-Carlin Student Award at the 2015 IEEE $15^{\text {th }}$ Mediterranean Microwave Symposium 2015.

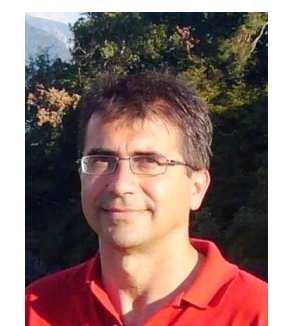

Alessandro Galli (S'91-M'96) received the Laurea degree in electronic engineering and the Ph.D. degree in applied electromagnetics from "La Sapienza University of Rome, Rome, Italy. Since 1990, he has been with the Department of Electronic Engineering (now Department of Information Engineering, Electronics and Telecommunications, DIET), "La Sapienza University of Rome. In 2000, he became an Assistant Professor, and in 2002 an Associate Professor with the Faculty of Engineering, 'La Sapienza University of Rome. In 2013, he won the National Scientific Qualification as a Full Professor in the sector of Electromagnetics. Dr. Galli has authored more than 300 papers on journals, books, and conferences. He is author of a patent for an invention concerning a type of microwave antenna. His research interests include theoretical and applied electromagnetics, mainly focused on modeling, numerical analysis, and design for antennas and passive devices at microwaves, millimeter waves, and terahertz: specific topics involve leaky waves, periodic and multilayered printed structures, metamaterials, and graphene. He is active also in geoelectromagnetics, bioelectromagnetics, and microwave plasma heating for alternative energy sources. In 2014, he was the general co-chair of the "European Microwave Week" (EuMW), the most important conference event in the electromagnetic area at European level. He was elected as the Italian representative of the Board of Directors of the "European Microwave Association" (EuMA), the main European Society of electromagnetics, for the 2010-2012 triennium and then re-elected for the 2013-2015 triennium. Since its foundation in 2012, he is the coordinator of the "European Courses on Microwaves" (EuCoM), the first European educational institution on microwaves. He is also a member of the "European School of Antennas" (ESoA). He is a member of the leading scientific societies of electromagnetics, and an Associate Editor of the International Journal of Microwave and Wireless Technologies (Cambridge University Press). He was the recipient of various grants and prizes for his research activity: he won in 1994 the "Barzilai Prize" for the best scientific work of under-35 researchers at the "10th National Meeting of Electromagnetics" and, in 1994 and in 1995, the "Quality Presentation Recognition Award" at the International Microwave Symposium by the "Microwave Theory and Techniques" (MTT) Society of the "Institute of Electrical and Electronics Engineering" (IEEE). 


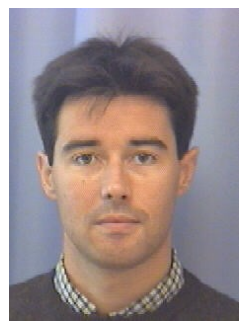

Ronan Sauleau (M'04-SM'06) graduated in electrical engineering and radio communications from the Institut National des Sciences Appliquées, Rennes, France, in 1995. He received the Agrégation degree from the Ecole Normale Supérieure de Cachan, France, in 1996, and the Doctoral degree in signal processing and telecommunications and the "Habilitation à Diriger des Recherches" degree from the University of Rennes 1, France, in 1999 and 2005, respectively. He was an Assistant Professor and Associate Professor at the University of Rennes 1, between September 2000 and November 2005, and between December 2005 and October 2009, respectively. He has been appointed as a full Professor in the same University since November 2009. His current research fields are numerical modeling (mainly FDTD), millimeter-wave printed and reconfigurable (MEMS) antennas, substrate integrated waveguide antennas, lens-based focusing devices, periodic and non-periodic structures (electromagnetic bandgap materials, metamaterials, reflectarrays, and transmitarrays) and biological effects of millimeter waves. He has been involved in more than 30 research projects at the national and European levels and has cosupervised 14 post-doctoral fellows, $28 \mathrm{PhD}$ students and 43 master students. He has received ten patents and is the author or coauthor of more than 175 journal papers and 380 publications in international conferences and workshops. He has shared the responsibility of the research activities on antennas at IETR in 2010 and 2011. He is now co-responsible for the research Department 'Antenna and Microwave Devices' at IETR and is deputy director of IETR. Prof. Sauleau received the 2004 ISAP Conference Young Researcher Scientist Fellowship (Japan) and the first Young Researcher Prize in Brittany, France, in 2001 for his research work on gain-enhanced FabryPerot antennas. In September 2007, he was elevated to Junior member of the "Institut Universitaire de France". He was awarded the Bronze medal by CNRS in 2008. He was the co-recipient of several international conference awards with some of his students (Int. Sch. of BioEM 2005, BEMS 2006, MRRS 2008, E-MRS 2011, BEMS 2011, IMS 2012, Antem 2012, BioEM 2015). He served as a guest editor for the IEEE Antennas Propogat. Special Issue on "Antennas and Propagation at $\mathrm{mm}$ and sub $\mathrm{mm}$ waves".

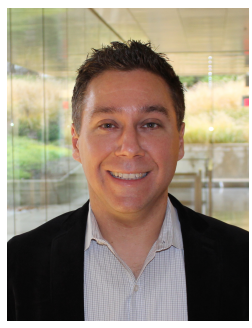

Anthony Grbic (S'00-M'06-SM'14) received the B.A.Sc., M.A.Sc., and Ph.D. degrees in electrical engineering from the University of Toronto, Canada, in 1998, 2000, and 2005, respectively. In January 2006, he joined the Department of Electrical Engineering and Computer Science, University of Michigan, Ann Arbor, where he is currently an Associate Professor. His research interests include engineered electromagnetic structures (metamaterials, metasurfaces, electromagnetic band-gap materials, frequency selective surfaces), plasmonics, antennas, analytical electromagnetics/optics, microwave circuits and wireless power transmission systems. Dr. Grbic received an AFOSR Young Investigator Award as well as an NSF Faculty Early Career Development Award in 2008. In January 2010, he was awarded a Presidential Early Career Award for Scientists and Engineers. In 2011, he received an Outstanding Young Engineer Award from the IEEE Microwave Theory and Techniques Society, a Henry Russel Award from the University of Michigan, and a Booker Fellowship from the United States National Committee of the International Union of Radio Science. In 2012, he was the inaugural recipient of the Ernest and Bettine Kuh Distinguished Faculty Scholar Award in the Department of Electrical and Computer Science, University of Michigan. Anthony Grbic served as Technical Program Co-Chair for the 2012 IEEE International Symposium on Antennas and Propagation and USNC-URSI National Radio Science Meeting (AP-S/USNC-URSI). He was an Associate Editor for IEEE Antennas and Wireless Propagation Letters from 2010 to 2015. He is currently Vice Chair of AP-S Technical Activities, Trident Chapter, IEEE Southeastern Michigan section. He will serve as a Topic CoChair for the 2016 AP-S/USNC-URSI.

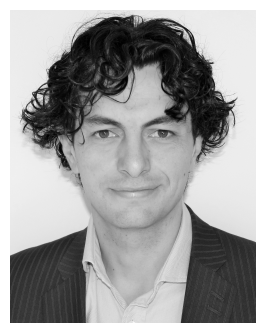

Mauro Ettorre (M'08-SM'15) was born in Tricarico, Matera, Italy, in 1979. He received the Laurea (summa cum laude) degree in telecommunication engineering and the Ph.D. degree in electromagnetics from the University of Siena, Siena, Italy, in 2004 and 2008, respectively. During his Master's degree studies, he spent 5 months with the Technical University of Denmark (DTU), Lyngby, Denmark. Part of his Ph.D. has been developed at the Defence, Security and Safety Institute of the Netherlands Organization for Applied Scientific Research (TNO), The Hague, The Netherlands, where afterward he worked as Antenna Researcher. ¿From 2008 to 2010, he was a Postdoctoral Fellow with the Institut d'Electronique et de Télécommunications de Rennes (IETR), Université de Rennes 1, Rennes Cedex, France. From 2010 to 2011, he was a Visiting Scholar at the Radiation Laboratory, Department of Electrical Engineering and Computer Science, University of Michigan, Ann Arbor, MI, USA. Since October 2010, he has been appointed as permanent CNRS Researcher with the IETR. His research interests include the analysis and design of leaky-wave antennas, periodic structures, compact planar antennas, near-field focusing techniques, and wireless power transmission systems. Dr. Ettorre was the recipient of the Young Antenna Engineer Prize during the 30th ESA Antenna Workshop 2008 in Noordwijk, The Netherlands. 